\title{
Cardiac neural crest orchestrates remodeling and functional maturation of mouse semilunar valves
}

\author{
Rajan Jain,1,2 Kurt A. Engleka,,1,2 Stacey L. Rentschler,,1,2 Lauren J. Manderfield,1,2 \\ Li Li, 1,2 Lijun Yuan,, 1,2,3 and Jonathan A. Epstein ${ }^{1,2}$
}

1Department of Cell and Developmental Biology and 2Division of Cardiology, Penn Cardiovascular Institute, University of Pennsylvania School of Medicine, Philadelphia, Pennsylvania, USA. ${ }^{3 T a n g d u}$ Hospital, Fourth Military Medical University, Xi'an, China.

\begin{abstract}
Congenital anomalies of the aortic valve are common and are associated with progressive valvular insufficiency and/or stenosis. In addition, aneurysm, coarctation, and dissection of the ascending aorta and aortic arch are often associated conditions that complicate patient management and increase morbidity and mortality. These associated aortopathies are commonly attributed to turbulent hemodynamic flow through the malformed valve leading to focal defects in the vessel wall. However, numerous surgical and pathological studies have identified widespread cystic medial necrosis and smooth muscle apoptosis throughout the aortic arch in affected patients. Here, we provide experimental evidence for an alternative model to explain the association of aortic vessel and valvular disease. Using mice with primary and secondary cardiac neural crest deficiencies, we have shown that neural crest contribution to the outflow endocardial cushions (the precursors of the semilunar valves) is required for late gestation valvular remodeling, mesenchymal apoptosis, and proper valve architecture. Neural crest was also shown to contribute to the smooth muscle layer of the wall of the ascending aorta and aortic arch. Hence, defects of cardiac neural crest can result in functionally abnormal semilunar valves and concomitant aortic arch artery abnormalities.
\end{abstract}

\section{Introduction}

Early stages of cardiac valve development have been extensively studied and include a well-recognized example of epithelial-mesenchymal transformation (EMT) in which endothelial cells underlying the primitive endocardial cushions respond to extracellular signals to invade the underlying matrix, change shape, and proliferate. This process of EMT results in relatively bulky and cellular endocardial cushions by mid-gestation. Subsequently, endocardial cushions remodel to form the thin valve leaflets that prevent reversal of blood flow in the mature heart. The signals and cellular events that mediate valve remodeling are poorly characterized, although apoptosis and alterations in extracellular matrix production have been described (1-5).

Semilunar valve development is distinguished from atrioventricular valve development by the infiltration of migrating neural crest, which orchestrates important aspects of outflow tract septation and aortic arch artery remodeling $(6,7)$. A subpopulation of cardiac neural crest cells differentiate into vascular smooth muscle cells that populate the walls of the ascending aorta, aortic arch, and head vessels, and defects of neural crest cells in animal models produce coarctation and interruption of the aortic arch and a wide range of related outflow tract and aortic arch artery defects (7-9). Despite abundant contributions of neural crest to the mesenchyme of the outflow tract endocardial cushions during midgestation, few neural crest derivatives are present in the mature semilunar valve leaflets (10).

Cardiac neural crest cells delaminate from the dorsal neural tube at approximately E8.5 in the mouse and migrate through the pharyngeal arches on their way to the forming heart $(10,11)$.

Conflict of interest: The authors have declared that no conflict of interest exists. Citation for this article: J Clin Invest. 2011;121(1):422-430. doi:10.1172/JCI44244.
Before entering the cardiac outflow tract at approximately E10, neural crest is in close apposition to second heart field mesoderm (12). Second heart precursors are characterized by expression of Islet 1 and are labeled by transgenic mice that utilize a specific "anterior heart field" (AHF) enhancer of the Mef2c locus $(13,14)$. Second heart precursors contribute primarily to myocardium in the right ventricle and outflow tract and to some smooth muscle and endothelial derivatives $(13,14)$. We have recently shown that defects in Notch signaling within second heart precursors result in cardiac defects reminiscent of those seen in humans with Alagille syndrome, which can be caused by mutations in Notch signaling components (15-17). Our data suggested that Notch signaling in the second heart field mediates interactions with the migrating cardiac neural crest that are responsible for appropriate outflow tract development. Interestingly, Alagille patients also display semilunar valve abnormalities (18). Notch mutations and copy number variations have been linked to tetralogy of Fallot, which is characterized by a dysmorphic pulmonic valve in addition to an overriding aorta, right ventricular hypertrophy, and ventricular septal defects $(19,20)$. NOTCH1 mutations have been associated with bicuspid aortic valve disease in humans without underlying Alagille syndrome or tetralogy of Fallot (21-23).

Bicuspid aortic valve disease is among the most common of congenital defects, affecting $1 \%-2 \%$ of the population (24). Bicuspid valves are characterized by the presence of only 2 complete commissures (though an incomplete third commissure is often present) and unequally sized leaflets (5). Aortic valve abnormalities are associated with aneurysms of the ascending aorta, ventricular septal defects, aortic coarctation, and dissection of the carotid and vertebral arteries, which are not all easily attributed to secondary hemodynamic effects of valvular irregularities (25-27). Intriguingly, craniofacial defects are also associated with bicuspid aortic valve, suggesting an 

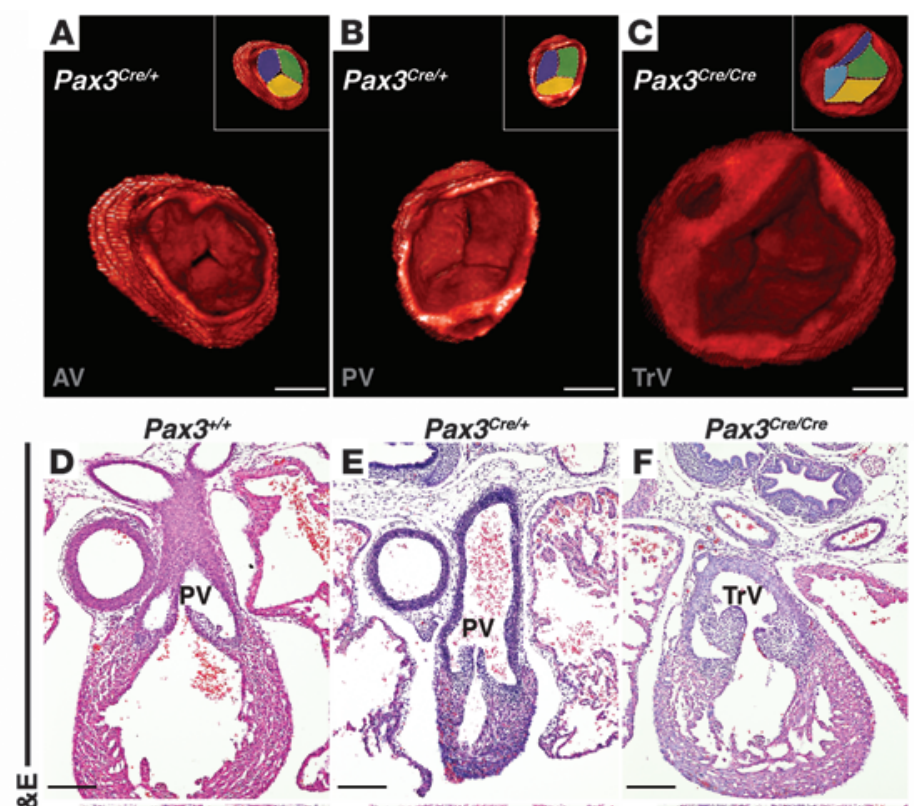

$\mathrm{Pax}^{\text {+/+ }}$ Pax3 $3^{\mathrm{Cre} / \mathrm{t}}$ $\operatorname{Tr}$

Pax3 ${ }^{\text {Cre/cre }}$

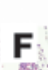

ination
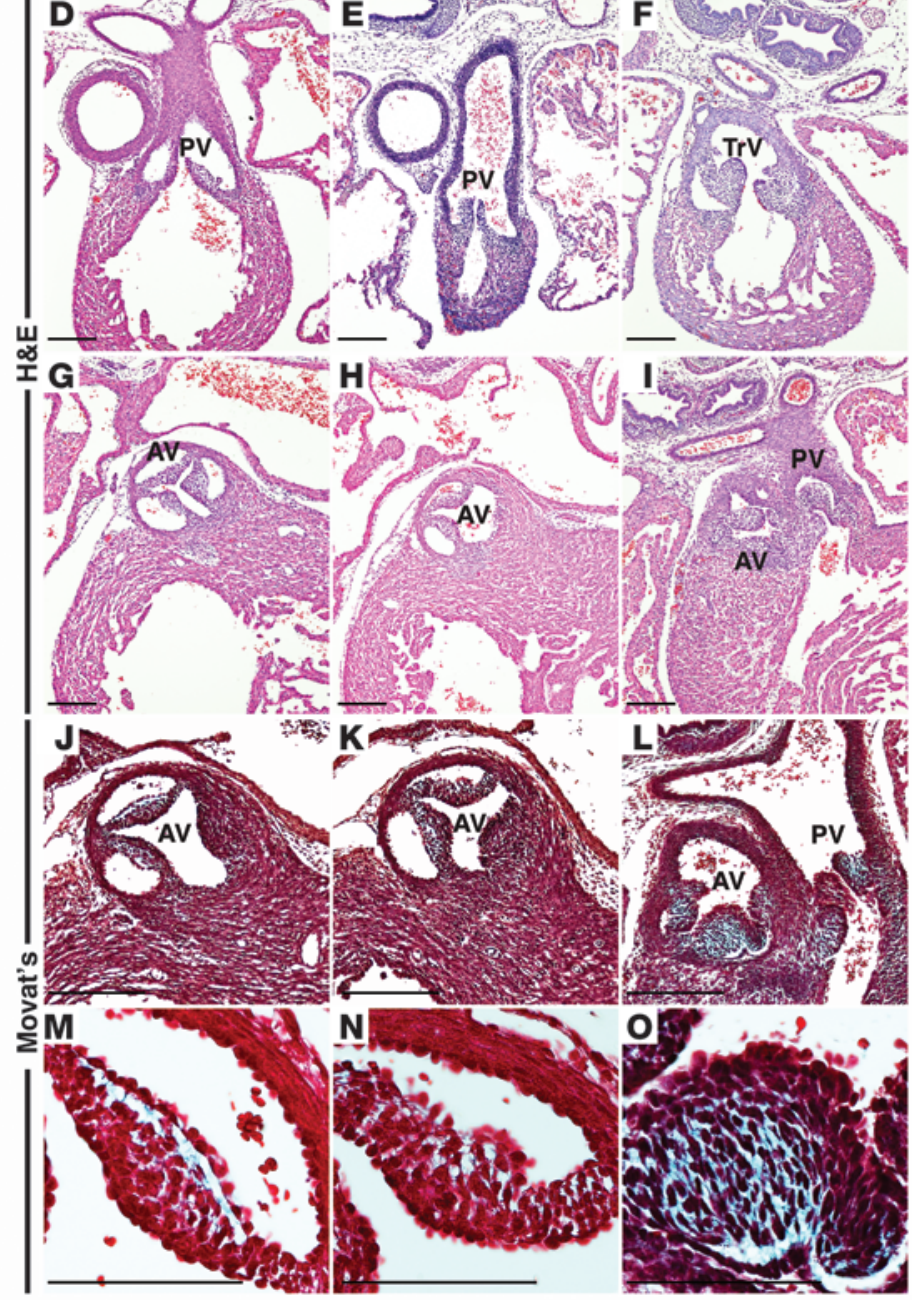

underlying relationship to neural crest (25), which contributes to craniofacial mesenchyme. Furthermore, numerous pathological studies have demonstrated noninflammatory degeneration of neural crestderived smooth muscle cells in the ascending aorta and aortic arch of patients with bicuspid aortic valves, even those without aneurysm formation, which is often characterized as cystic medial necrosis (28-31). Nevertheless, experimental evidence to support a common underlying developmental mechanism to explain the association of aortic valve and associated aortopathy has been lacking.

In order to explore the role of neural crest and related tissuetissue interactions during late stages of semilunar valve remodeling, we examined mice with genetic defects restricted to neu-

\section{Figure 1}

Loss of Pax3 results in abnormal semilunar valve leaflets. 3D reconstruction of OPT images of E16.5 $\mathrm{Pax} 3 \mathrm{Cre} /+$ control embryos demonstrates a trileaflet aortic $(\mathbf{A})$ and pulmonic $(\mathbf{B})$ valve, each

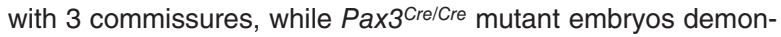
strate abnormal semilunar valve leaflets. The mutant depicted in $\mathbf{C}$ displays a truncal valve with 4 leaflets. Insets in $\mathbf{A}-\mathbf{C}$ represent identical images with each leaflet pseudocolored. Cross-sectional $\mathrm{H} \& \mathrm{E}$ images through the pulmonic valve leaflets of E16.5 $\mathrm{Pax} 3^{+/+}$ and $\mathrm{Pax} 3_{\mathrm{Cre} /+}$ embryos (D and $\left.\mathbf{E}\right)$ and a Pax3Cre/Cre littermate (F) show thickened leaflets in the mutant. Cross-sectional H\&E images through the aortic valve leaflets of E16.5 $\mathrm{Pax} 3^{+/+}$and $\mathrm{Pax} 3^{\mathrm{Cr} /+}$ embryos ( $\mathbf{G}$ and $\mathbf{H})$ and a $P a x 3^{C r e / C r e ~ l i t t e r m a t e ~(I) ~ s h o w ~ t h i c k e n e d, ~}$ unequally sized leaflets in the mutant. Examples of a truncal valve (F) and double-outlet right ventricle (I) are shown. Modified Movat's Pentachrome staining reveals extracellular matrix deposition (blue) in E16.5 control semilunar valve leaflets ( $\mathbf{J}$ and $\mathbf{K}$ ) and Pax3 Cre/Cre embryos (L), which show an increase in extracellular matrix compared with control leaflets. Higher magnification images of $\mathbf{J}-\mathbf{L}$ are shown as $\mathbf{M}-\mathbf{O}$. AV, aortic valve; PV, pulmonic valve; $\mathrm{TrV}$, truncal valve. Brightness and contrast of OPT images were adjusted using OsiriX software. Scale bars: $100 \mu \mathrm{m}$.

ral crest and mice with second heart field defects causing secondary neural crest abnormalities. We show that loss of Pax3 in neural crest leads to dysmorphic and thickened semilunar valves that are functionally incompetent. These abnormal valves display an increase in extracellular matrix deposition and a deficiency of mesenchymal apoptosis, which normally accompanies late stage remodeling and valvular thinning. Furthermore, inhibition of Notch signaling that is restricted to the second heart field results in secondary abnormalities of neural crest patterning and semilunar valve abnormalities that are virtually identical to those seen in $\mathrm{Pax} 3$ mutants. These results uncover what we believe is a novel and critical role for cardiac neural crest in the formation of the semilunar valves and illustrate the importance of coordinate tissue-tissue interactions during cardiac development.

\section{Results}

Loss of Pax 3 results in abnormal semilunar valve morphology. Pax3 is expressed by premigratory neural crest, and Pax3 mutants display well-characterized defects of multiple neural crest derivatives $(11,32,33)$. For example, Splotch mutant embryos on a C57BL/ 6 genetic background lacking Pax3 succumb by E13.5 and display complete failure of outflow tract septation, deficiency of premigratory neural crest expansion, and substantial abnormalities of postmigratory cardiac neural crest patterning and function (34-36). Mid-gestation lethality has precluded examination of later stages or an investigation of the role of Pax3 in semilunar valve maturation. Therefore, we outcrossed Pax3 mutants onto a CD1 mixed genetic background and noted occasional survival of mutant embryos through late gestation, though we never observed any live-born mutant pups (of more than 100 litters examined derived from heterozygous crosses).

Optical projection tomography (OPT) of E16.5 WT or Pax $3^{\mathrm{Cre} /+}$ control embryos demonstrated trileaflet aortic and pulmonic valves with 3 complete commissures (Figure 1, A and B, and Supplemental Videos 1 and 2; supplemental material available online with this article; doi:10.1172/JCI44244DS1). However, $\mathrm{Pax} 3^{\mathrm{Cr} / \mathrm{Cre}}$ mutant embryos 


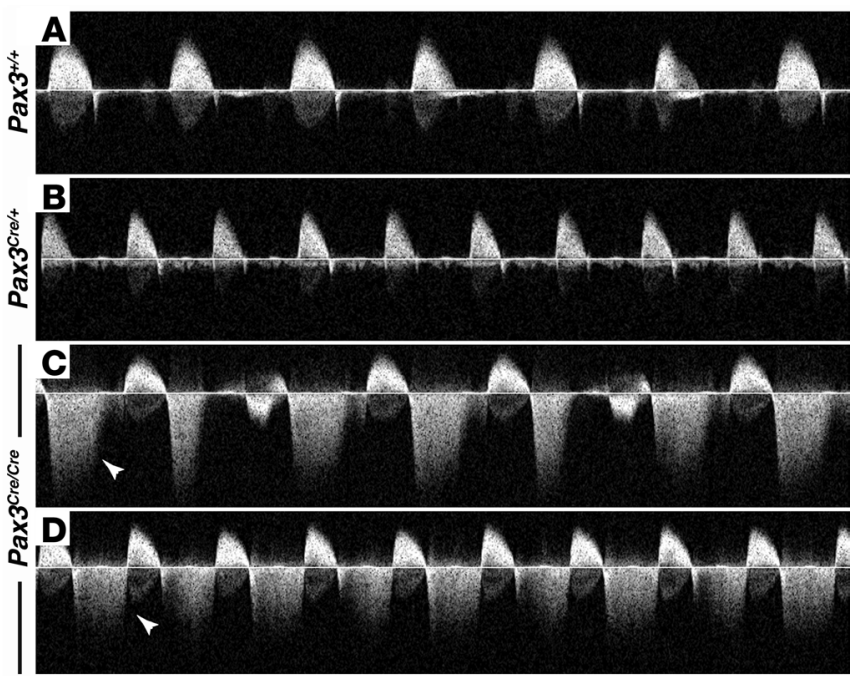

Figure 2

Loss of Pax3 results in aortic regurgitation. Pulse-wave Doppler analysis from the aorta of $\mathrm{E} 16.5 \mathrm{Pax}^{+/+}$and $\mathrm{Pax} 3^{\mathrm{Cre} /+}$ control embryos demonstrate no significant aortic regurgitation ( $\mathbf{A}$ and $\mathbf{B})$. Pax3 $\mathrm{Cre} / \mathrm{Cre}$ littermates demonstrate at least moderate-severe aortic insufficiency (arrowhead in C and D). Arrowheads point to regurgitant flow in mutant embryos.

demonstrated large, thickened valve leaflets resulting in a lack of coaptation (Figure 1C and Supplemental Video 3). Frequently, only 2 complete commissures were visualized, consistent with a bicuspid valve anomaly. Using OPT data, we quantified the average semilunar valve leaflet volume from E16.5 mutant and control embryos. The average semilunar valve leaflet volume of $\mathrm{Pa} \times 3^{\mathrm{Cre} / \mathrm{Cre}}$ mutant embryos was 2.6 times larger than that of control littermates $\left(4,597,608 \pm 823,585 \mu \mathrm{m}^{3}[n=3\right.$ embryos, 4 valves] versus $1,791,527 \pm 217,029 \mu \mathrm{m}^{3}[n=3$ embryos, 6 valves]; $P=0.0029)$.

Histological analysis revealed semilunar valves with thin, crisp edges in both $\mathrm{Pax}^{+/+}$and $\mathrm{Pa} \times 3^{\mathrm{Cre} /+}$ control embryos at E16.5, while $\mathrm{Pax} 3^{\mathrm{Cre} / \mathrm{Cre}}$ littermates displayed severely dysmorphic and thickened semilunar valve leaflets of unequal size (Figure 1, D-I). The atrioventricular valves, which do not receive neural crest contribution, appeared normal in these embryos (Supplemental Figure 1). More detailed analysis revealed the normal trilaminar architecture and organized cellularity in control embryo aortic valve leaflets, while mutant embryos displayed an increase of extracellular matrix in the mutant valve leaflets (Figure 1, J-O).

Loss of Pax 3 results in abnormal semilunar valve function. We assessed semilunar valve function by intrauterine echocardiography at E16.5. Embryos from 3 litters of timed pregnant female Pax $3^{\mathrm{Cr} /+}$ mice, impregnated by a Pax $3^{\mathrm{Cre} /+}$ male, were examined by pulsewave Doppler analysis of the aorta for evidence of flow reversal. Studies were performed prior to embryo genotyping, and the examinations and image interpretation were performed in a blinded fashion. All $\mathrm{Pax}^{+/+}(11 / 11)$ and $\mathrm{Pa} 3^{\mathrm{Cre} /+}$ embryos $(16 / 16)$ demonstrated absence of detectable regurgitant flow beyond trace amounts associated with valve closure (Figure 2, A and B). All

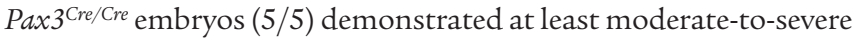
aortic insufficiency, characterized by a regurgitant jet present through the entirety of diastole (Figure 2, C and D). Aortic insufficiency has been noted in some studies to be the most prevalent valvular abnormality in humans with a bicuspid aortic valve (37).
Semilunar valve defects in Notch second heart field mutants. We investigated the expression of neural crest markers in control and

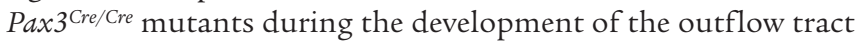
cushions. Sema3C is robustly expressed by postmigratory cardiac neural crest (38). Control embryos demonstrated 2 clusters of neural crest cells in the outflow tract cushions, as noted previously $(7,10,39)$, at E11.5 by in situ hybridization (Figure 3A). These cells are at the leading edge of the columns of neural crest

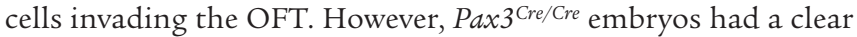
reduction in the Sema3C expression domain in the outflow tract cushions (Figure 3B). The mutant embryos maintained myocardial Sema3C expression within the myocardial wall of the outflow tract, demonstrating sensitive detection of Sema3C expression by in situ hybridization in mutant embryos. These data are consistent with both a reduction in the number of cardiac neural crest cells and altered migration characteristics as previously reported in Splotch mutants $(34,36,40)$.

We have recently shown that inhibition of Notch signaling in the second heart field results in complex outflow tract and pharyngeal arch artery abnormalities that are strikingly reminiscent of those resulting from neural crest defects (15). As second heart field precursors migrate through the pharyngeal mesenchyme, they are located in close proximity to migrating neural crest cells. Therefore, we have postulated that cardiovascular defects in the Notch second heart field mutants are due, in part, to abnormal neural crest patterning (15). Hence, we sought to examine the semilunar valves in second heart field Notch mutants to determine whether they showed abnormalities similar to those seen in Pax3 neural crest mutants.

Disruption of Notch signaling in the second heart field was accomplished by cre-inducible expression of a truncated form of the mastermind-like protein DNMAML, using 2 distinct second heart field cre mice, Islet $1^{\mathrm{Cre} /+}$ and Mef2c-AHF-Cre. All 4 mammalian Notch receptors interact with mastermind-like proteins after ligand-mediated activation and translocation to the nucleus. DNMAML is a well-characterized truncated form of mastermind-like protein that acts as a specific and effective Notch inhibitor by binding to the Notch intracellular domain and preventing recruitment of coactivators. We used mice in which DNMAML (knocked into the constitutively active Rosa26 locus) is expressed in a tissue-specific manner after activation by cre recombinase (41). We confirmed that activation of DNMAML in the second heart field by either Islet $1^{\text {Cre/+ }}$ or Mef2c-AHF-Cre resulted in abnormal neural crest patterning in outflow tract endocardial cushions. Islet ${ }^{\text {Cre/+}} ; D N M A M L$ and Mef2c-AHF-Cre;DNMAML mutant embryos showed a paucity of Sema3C expression in the outflow cushions at E11.5 (Figure 3, C and D). We confirmed these results with a second marker of postmigratory neural crest, PlexinA2. PlexinA2 expression was decreased in Pax 3 Cre/Cre mice, Islet $1^{\mathrm{Cre} /+}$;DNMAML mice, and Mef2c-AHF-Cre;DNMAML mutants (Figure 3, E-H). Similarly, AP2 $\alpha$ expression (a third marker of postmigratory neural crest) was deficient in $M e f 2 c$ AHF-Cre;DNAMAML mutants (Figure 3, I and J).

Abnormal neural crest patterning in Notch second heart field mutants suggests that Notch is a mediator of signaling between the second heart field and migrating neural crest. We detected apposition of migrating neural crest derivatives and Islet1-expressing pharyngeal mesoderm as early as E9.5 within the ventral pharynx. $P a x 3^{\mathrm{Cr} /{ }^{+}} ; Z / E G$ embryos (in which the $Z / E G$ allele serves as a cre-reporter allele that activates expression of 


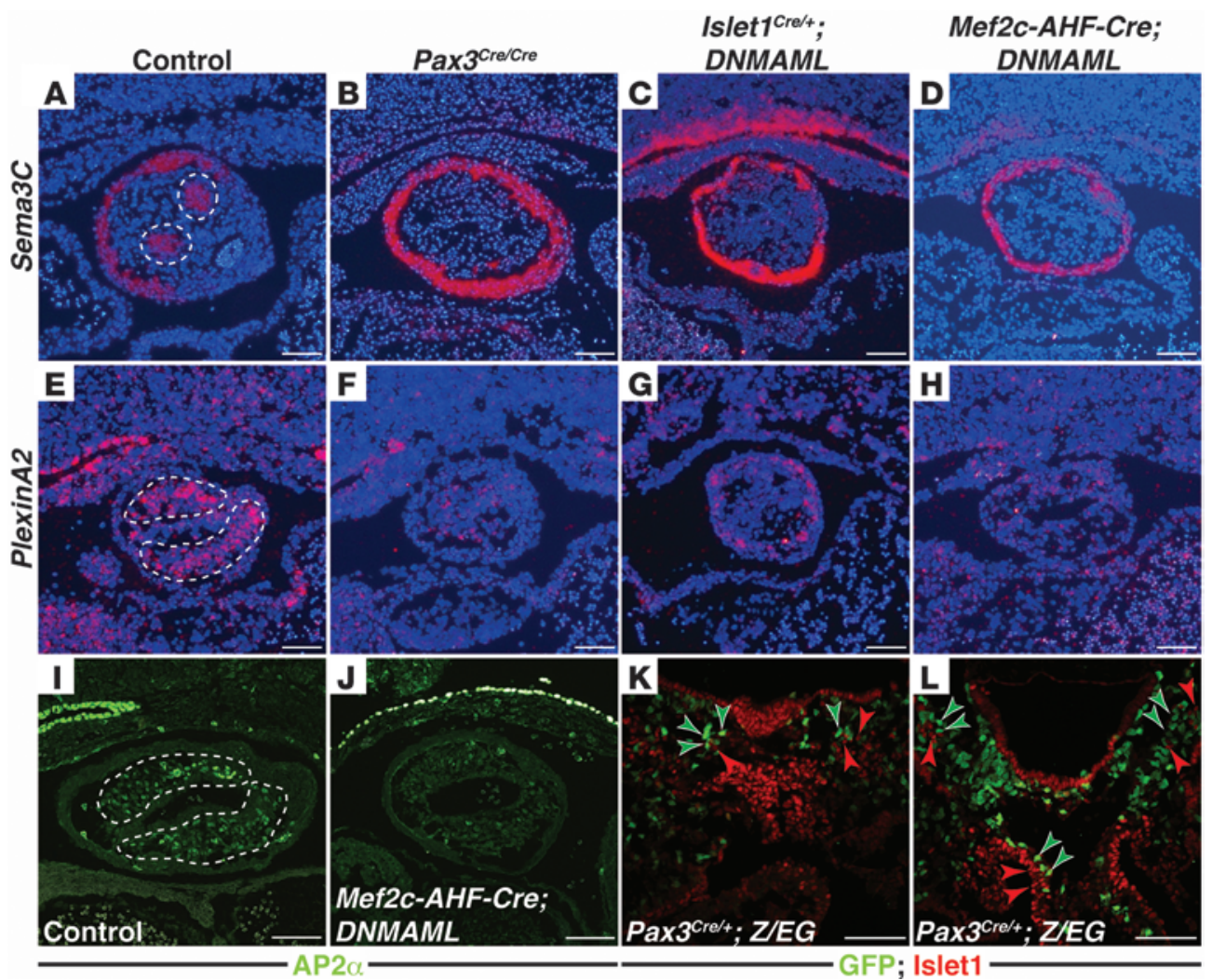

Figure 3

Abnormal neural crest migration in neural crest and second heart field mutants. In situ hybridization for neural crest marker Sema3C of E11.5 coronal sections through the outflow tract cushions of control (WT) (A), Pax3Cre/Cre (B), Islet1 cre/+;DNMAML (C), and Mef2c-AHF-Cre;DNMAML (D) embryos. In situ hybridization for neural crest marker PlexinA2 of E11.5 coronal sections of control (WT) (E), Pax3 Cre/Cre (F), Islet 1 Cre/+;DNMAML $(\mathbf{G})$, and Mef2c-AHF-Cre;DNMAML (H) embryos. AP2 $\alpha$ immunohistochemistry of coronal sections of the outflow tract cushions of E10.5 control (WT) (I) and Mef2c-AHF-Cre;DNMAML (J) embryos. Dotted lines in A, E, and I enclose regions occupied by neural crest cells expressing Sema3C, PlexinA2, and AP2 $\alpha$, respectively. GFP and Islet1 immunohistochemistry of cross sections through the outflow tract and pharyngeal mesenchyme of E9.5 Pax3 ${ }^{C r e /+} ; Z / E G$ embryos (K and L). GFP-positive (neural crest derivatives) and Islet1-positive (second heart field) cells that are in close apposition to one another are highlighted with green (GFP) or red (Islet1) arrowheads. Scale bars: $100 \mu \mathrm{m}$.

GFP in response to cre-mediated recombination; ref. 42) demonstrated neural crest derivatives adjacent to clusters of Islet1expressing second heart field cells (Figure 3, K and L). This represents a potential site for paracrine signaling between the 2 cell populations.

OPT analysis and subsequent $3 \mathrm{D}$ reconstruction of E17.5 control embryos demonstrated trileaflet pulmonic and aortic valves (Figure 4, A and B, and Supplemental Videos 4 and 5), while Mef2cAHF-Cre;DNMAML littermate mutants demonstrated dysmorphic, thickened valve leaflets (Figure 4C and Supplemental Video 6). Mutants frequently displayed unequally sized leaflets with bicuspid-like anomalies (note the pulmonic valve with 2 commissures in Figure 4C and Supplemental Video 6). Quantification of the average semilunar valve leaflet volume of E17.5 embryos confirmed enlargement of mutant leaflets. Mef2c-AHF-Cre;DNMAML semilunar valve leaflets were 2.2 times larger than those of WT littermates $\left(6,318,525 \pm 158,618 \mu \mathrm{m}^{3}[n=3\right.$ embryos, 5 valves $]$ versus $2,906,227 \pm 275,426 \mu \mathrm{m}^{3}$ [ $n=3$ embryos, 5 valves]; $P=0.0001$ ).

Upon histological analysis, control embryos demonstrated thin, delicate semilunar valve leaflets (Figure 4, D and E). In contrast, both Islet ${ }^{\mathrm{Cre} /+}$;DNMAML (Figure 4F) and Mef2c-AHF-Cre;DNMAML (Figure 4, G and $\mathrm{H}$ ) mutants demonstrated abnormal semilunar valve leaflets, strikingly similar to the Pax3 $\mathrm{Cre} / \mathrm{Cre}$ animals. Outflow valve leaflets were abnormal irrespective of associated outflow tract and aortic arch abnormalities such as truncus arteriosus or double-outlet right ventricle. The control leaflets possessed normal trilaminar extracellular matrix organization (Figure 4, I and J), but the mutants exhibited an excess of extracellular matrix (Figure 4, K-M). Atrioventricular valve leaflets were unaffected (Supplemental Figure 1).

Intrauterine echocardiography of E17.5 Islet $1^{\text {Cre } /+}$;DNMAML or Mef2c-AHF-Cre;DNMAML embryos was utilized to assess valve competency. All 6 control (littermate WT) embryos demonstrated trivial or no aortic insufficiency (Figure 5A). However, each of 4 Islet $1^{\text {Cre/+}}$;DNMAML embryos demonstrated at least moderateto-severe aortic insufficiency (Figure 5B). Likewise, 5 additional control (WT littermate) embryos examined were free of significant aortic insufficiency, while all 4 Mef2c-AHF-Cre;DNMAML mutant littermates demonstrated at least moderate-to-severe aortic insufficiency (Figure 5, C and D). A regurgitant jet present throughout diastole was defined as moderate-to-severe aortic insufficiency. Thus, semilunar valves of embryos in which Notch signaling is inhibited in the second heart field are morphologically and functionally abnormal. 

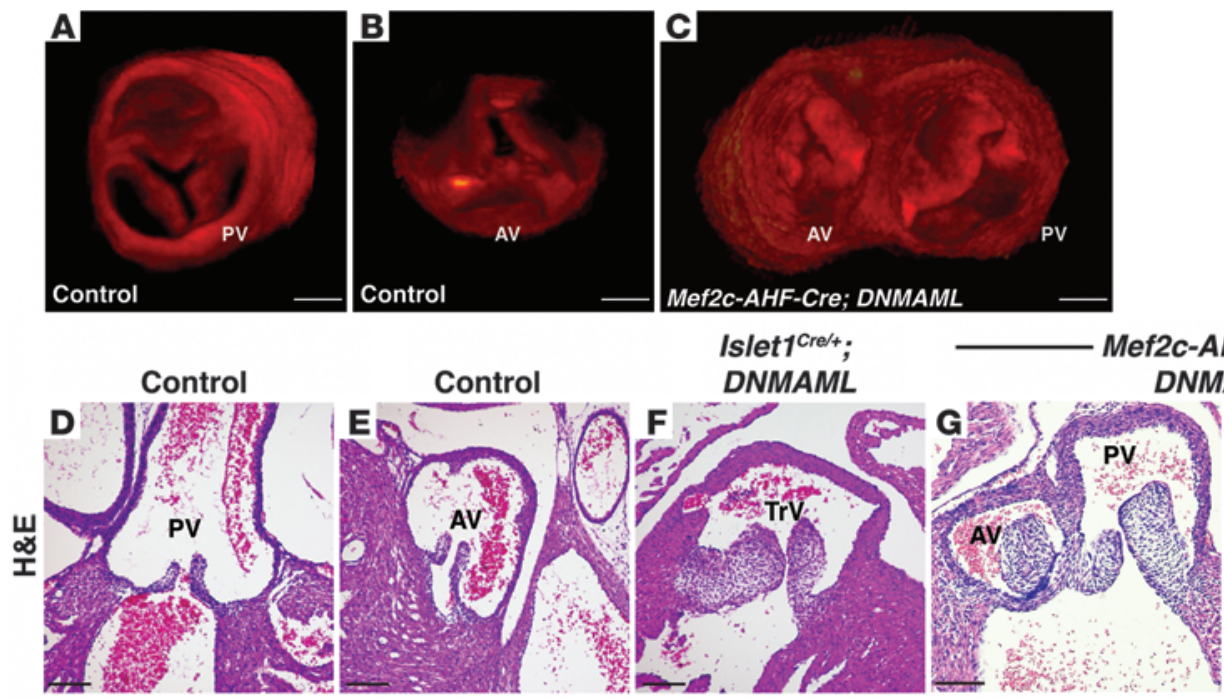

DNMAML
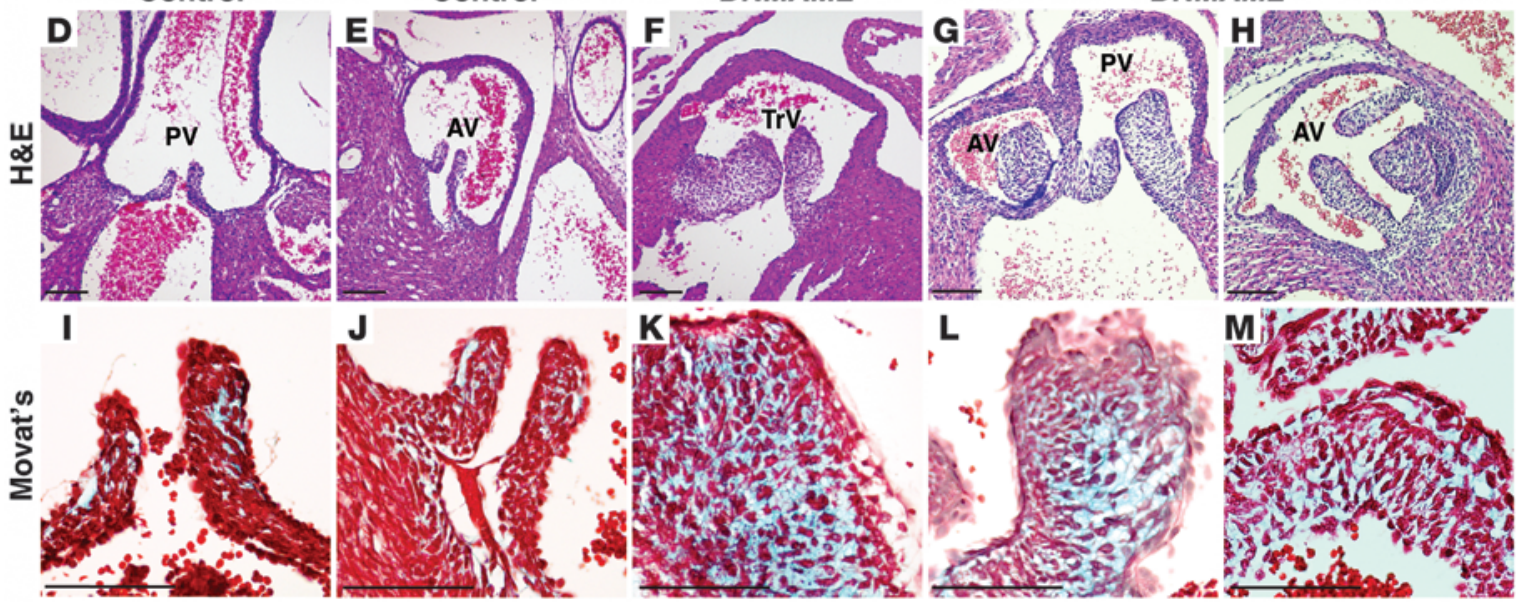

Figure 4

Notch inhibition in the second heart field results in dysmorphic semilunar valve leaflets. 3D reconstructions of OPT-generated images of E17.5 control (WT) pulmonic valve (A), aortic valve (B), and Mef2c-AHF-Cre;DNMAML semilunar valve leaflets (C). Note the thickened aortic valve leaflets and bicuspid pulmonic valve in the mutant (C), which demonstrates a double-outlet right ventricle. H\&E staining of cross sections of E17.5 control (WT) pulmonic (D) and aortic valves (E) demonstrates delicate, thin leaflets. However, Islet1 ${ }^{\text {Cre/t; }}$ DNMAML (F) and Mef2c-AHFCre;DNMAML semilunar valve leaflets $(\mathbf{G}$ and $\mathbf{H})$ are thickened and of unequal sizes. Modified Movat's Pentachrome staining of cross sections of E17.5 control (WT) pulmonic (I), aortic (J), Islet1 Cre/+;DNMAML (K), and Mef2c-AHF-Cre;DNMAML valve leaflets (L and $\mathbf{M})$. Mutant leaflets demonstrate an increase in extracellular matrix deposition. $\mathbf{F}$ and $\mathbf{K}$ show embryos with a persistent truncus arteriosus, and $\mathbf{G}, \mathbf{H}, \mathbf{L}$, and $\mathbf{M}$ show embryos with a double-outlet right ventricle. Brightness and contrast of OPT images were adjusted using OsiriX software. Scale bars: $100 \mu \mathrm{m}$.

Abnormal semilunar valves are associated with a deficiency of apoptosis and remodeling. Neural crest derivatives can be identified within the outflow tract endocardial cushions as early as E10.5, although few derivatives remain by E17.5 (Supplemental Figure 2). The developing semilunar valve leaflets retain substantial contribution of neural crest derivatives at E13.5, but by E16.5 there is a considerable decrease. By E17.5, the semilunar valve leaflets are nearly devoid of neural crest derivatives (Supplemental Figure 2). In neural crest mutants and in second heart field mutants with neural crest patterning defects, outflow endocardial cushions are initially hypocellular, at least in part due to a deficiency of neural crest mesenchymal derivatives (15). However, paradoxically, these mutants display hypercellular semilunar valves by late gestation (Figures 1 and 4). Since normal late remodeling of the semilunar valves is associated with a decrease in mesenchymal proliferation and an increase in apoptosis, we examined these parameters in normal and mutant embryos during late gestation (E16.5-E17.5).

$\mathrm{Pax}^{+/+}$and $\mathrm{Pax} 3^{\mathrm{Cre} /+}$ control semilunar valve leaflet mesenchyme exhibited $0.51 \%$ and $0.53 \% \mathrm{TUNEL}^{+}$cells, respectively $(n=3$ embryos for each genotype, 6495 and 7314 mesenchymal cells counted, respectively) at E16.5. However, littermate $\mathrm{Pax} 3^{\mathrm{Cre} / \mathrm{Cre}}$ embryos demonstrated a statistically significant defi- ciency of apoptosis $\left(0.17 \% \mathrm{TUNEL}^{+}\right.$cells, 8749 mesenchymal cells counted, $P<0.005$ versus either control genotype; Figure $6 \mathrm{~A}$ and Supplemental Figure 3). We did not detect a statistically significant difference in proliferation as assessed by the percentage of phospho-histone $\mathrm{H}^{+}$cells ( $\mathrm{Pax} 3^{\mathrm{Cre} /{ }^{+}}$: $0.40 \% \pm 0.20 \mathrm{vs}$. Pax3 $\left.{ }^{\text {Cre } / \text { Cre }: ~} 0.49 \% \pm 0.14, P=0.74\right)$. Likewise, E17.5 Mef2c-AHFCre+;DNMAML embryo semilunar valve leaflets displayed a statistically significant decrease in apoptosis when compared with control littermates $\left(0.38 \% \mathrm{TUNEL}^{+}\right.$cells in DNMAML control [ $n=3$ embryos, 15445 cells counted] versus $0.09 \% \mathrm{TUNEL}^{+}$cells in Mef2c-AHF-Cre;DNMAML [ $n=3$ embryos, 40707 cells counted, $P=0.0004$, Figure 6B and Supplemental Figure 3]), while no statistically significant change in phospho-histone $\mathrm{H} 3^{+}$cells was noted. Similar results were obtained when Islet $1^{\text {cre/+}} ;$ DNMAML embryos were compared with control littermates (data not shown). Hence, deficiency of neural crest contribution to the outflow endocardial cushions is characterized by an increase in extracellular matrix (Figures 1 and 4) and a deficiency of apoptosis (Figure 6) during late stages of valve remodeling. Our data suggest that neural crest cells in the semilunar valve leaflets initiate an apoptotic process of surrounding mesenchymal cells that contributes to valve remodeling. Consistent with this hypothesis, less than $5 \%$ of apoptotic nuclei counted in $\mathrm{Pax} 3^{\mathrm{Cre} /+} ; \mathrm{Z} / \mathrm{EG}$ 


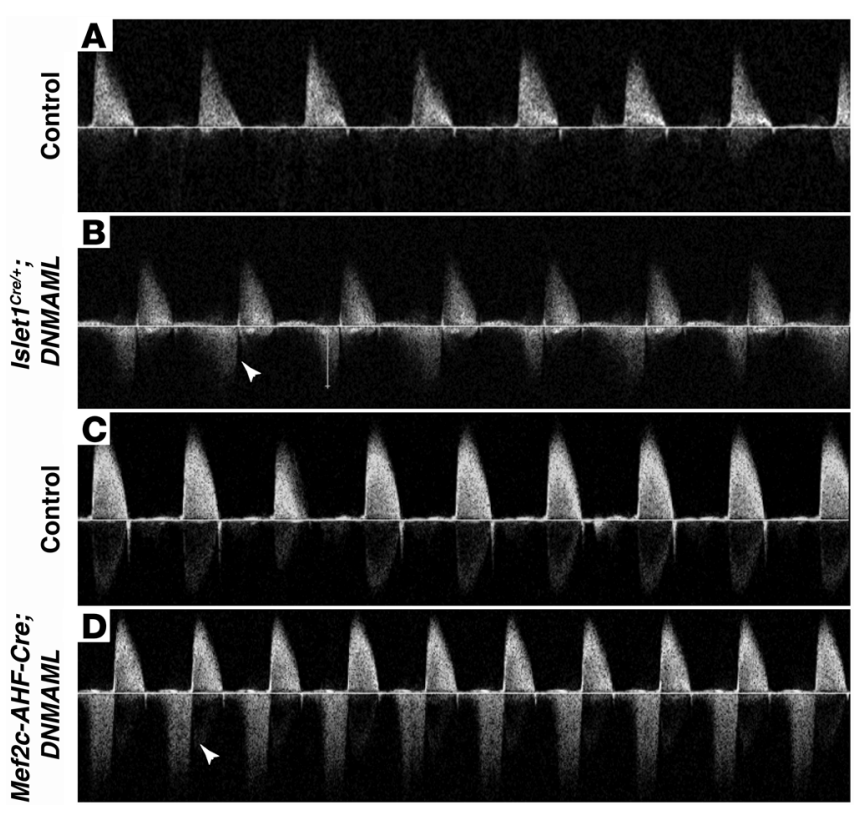

E16.5 semilunar valve leaflets were neural crest derivatives (3/68 nuclei counted), but our data do not exclude the possibility that they are second heart field derivatives.

\section{Discussion}

Our results demonstrate what we believe is a novel role for neural crest cells in semilunar valve leaflet maturation. During normal cardiogenesis, migrating neural crest and second heart field cells are in close proximity in the ventral pharynx and endocardial cushions (Figure 3, K and L). Our data suggest that instructive cues are exchanged between these cell fields and these cues are necessary for semilunar valve maturation. Specifically, we provide evidence that signals from the neural crest populating the outflow endocardial cushions mediate alterations in extracellular matrix production and promote apoptosis that characterizes late gestation semilunar valve leaflet remodeling (Figure 7). Deletion of Pax3 results in abnormal neural crest patterning and bicuspid-like valve leaflets, which are characterized by an increase in extracellular matrix and deficiency of late-gestation apoptosis. Notch inhibition in the second heart field results in abnormal neural crest cell patterning, and these mutants also display similarly dysmorphic semilunar valve leaflets, though we cannot exclude a direct effect on second heart field-derived mesenchyme. These results provide evidence that semilunar valve leaflet remodeling is dependent upon appropriate tissue-tissue

\section{Figure 5}

Inhibition of Notch signaling in the second heart field results in aortic regurgitation. Pulse-wave Doppler analysis from the aorta of E17.5 control (A), Islet1 Cre/+;DNMAML (B), control (C), and Mef2c-AHF-Cre;DNMAML (D) embryos. Control embryos are WT littermates of the respective mutants. Arrowheads point to regurgitant flow in mutant embryos.

interactions amongst the second heart field, neural crest, and valve mesenchyme. Though all our mutants display outflow tract defects, our data, in accord with others, suggest that semilunar valve leaflet maturation occurs late in gestation, while septation and patterning of the outflow tract occur at earlier time points (15). Nonetheless, we cannot exclude the possibility that the valve abnormalities we detect are secondary to or dependent upon the outflow defects.

Several studies have shown an association between NOTCH1 mutations and the presence of a bicuspid aortic valve. Garg et al. identified 2 families with multiple generations with congenital cardiac defects including bicuspid valve disease that were associated with mutations in NOTCH1, including R1108X resulting in a premature stop codon and $\mathrm{H} 1505 \mathrm{del}$ resulting in a frameshift mutation and truncated protein (21). Other studies have identified other NOTCH1 mutations associated with sporadic forms of bicuspid aortic valve, including those with thoracic aortic aneurysms $(22,23)$. These studies did not address the specific tissue in which Notch functions during semilunar valve maturation. Notch signaling regulates endothelial-mesenchymal transformation during endocardial cushion formation (43). However, our data suggest that abnormalities of Notch signaling in neural crest (9) or second heart field can also contribute to the development of abnormal semilunar valves. Our previous studies have shown that inhibition of Notch in the second heart field impairs Fgf8 signaling (15). Interestingly, engineered deficiencies in Fgf8 can also result in bicuspid aortic valve and vascular smooth muscle abnormalities of the great arteries $(44,45)$. Fgf8 expressed by second heart field mesoderm

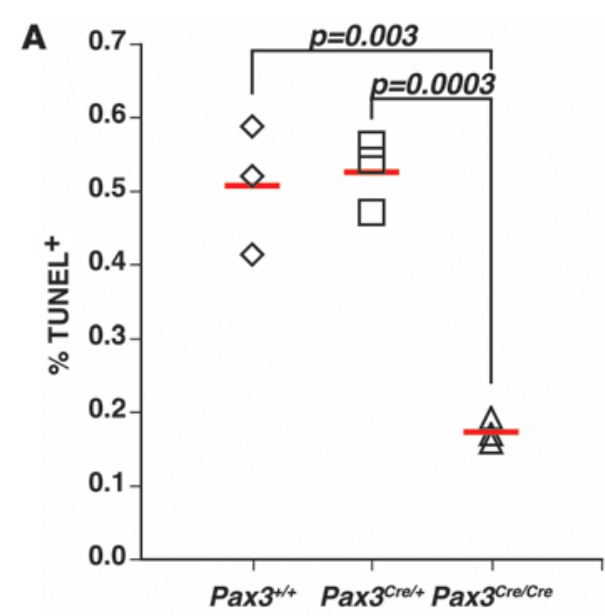

Figure 6

Loss of Pax3 or impaired second heart field Notch signaling results in diminished mesenchymal apoptosis in late gestation semilunar valve leaflets. Quantification of TUNEL+ cells (as a percentage of all DAPI ${ }^{+}$ mesenchymal cells) in E16.5 semilunar valve leaflets of Pax3 mutants (A). At least 6 sections were analyzed from each of 3 embryos for each genotype. Total number of cells counted are as follows: $P a \times 3^{+/+}, 6495$ cells; Pax3Cre/+, 7314 cells; and Pax3Cre/Cre, 8749 cells. E17.5 semilunar valve leaflets of control (WT) and Mef2c-AHF-Cre;DNMAML embryos (B). At least 8 sections were analyzed from each of 3 embryos for each genotype. Total number of cells counted are as follows: control, 15445 cells; and Mef2c-AHF-Cre;DNMAML, 40707 cells. Each point represents the percentage of TUNEL+ cells in an individual embryo. 


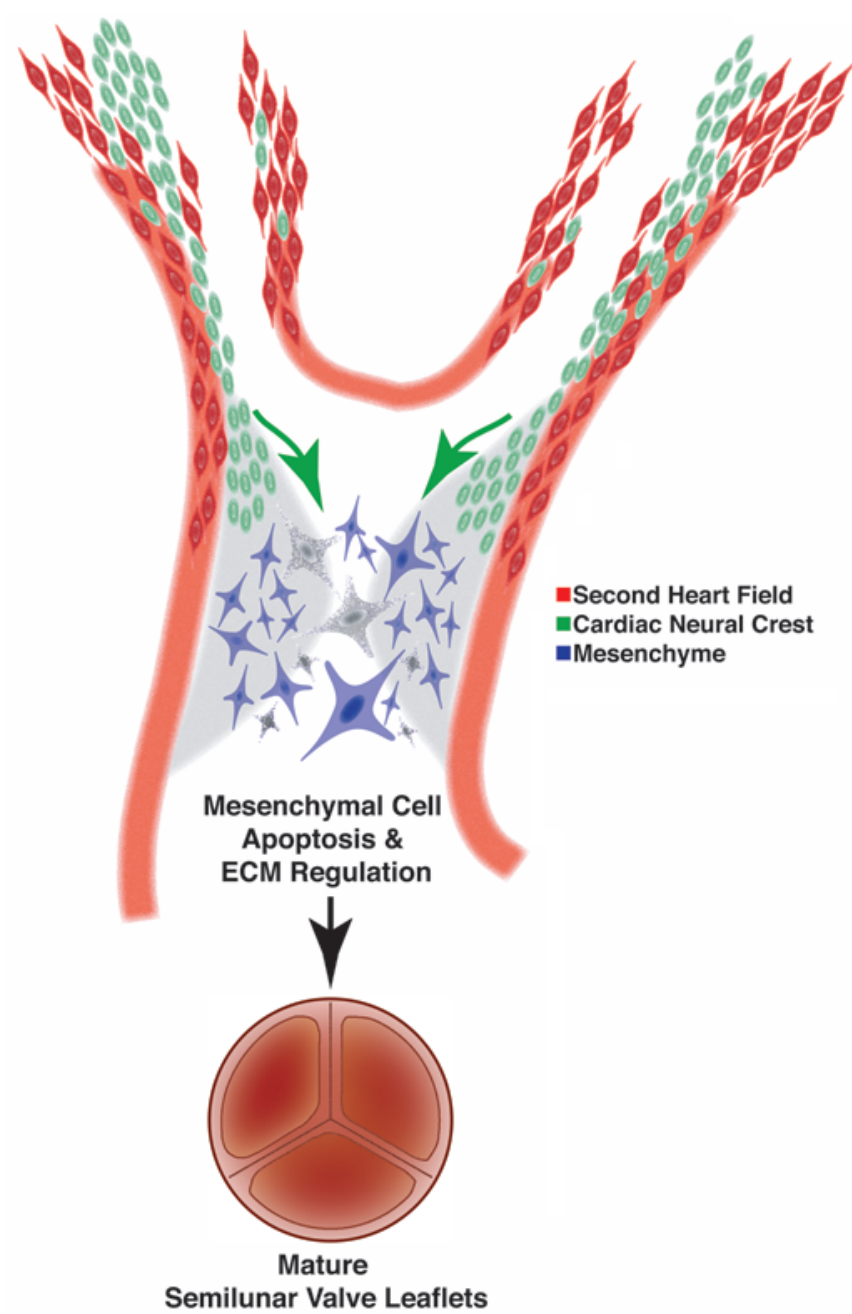

Figure 7

Model depicting the role of neural crest in semilunar valve development. Neural crest cells (green) are in close apposition to second heart field precursors (red) in the ventral pharynx during migration to the outflow endocardial cushions (light gray), where they provide instructive signals to orchestrate apoptosis (depicted as dark gray cells) and changes in extracellular matrix production during valve remodeling.

is likely to function in an autocrine fashion (46) and the signals that mediate crosstalk between the second heart field and neural crest remain to be fully elucidated. Further studies investigating the modulators of Notch and Fgf8 signaling in syndromic and nonsyndromic cases of bicuspid aortic valve will be of interest.

Multiple types of aortic arch malformations are associated with abnormal semilunar valves, including bicuspid aortic valve. The association between bicuspid aortic valve and aortic dissection and aneurysm is well documented, and interestingly, studies have shown that up to $60 \%$ of patients with aortic coarctation have a concomitant bicuspid aortic valve $(28,30,31,47)$. In addition, multiple cases of carotid and vertebral artery dissection in patients with bicuspid aortic valve have been reported (26). In some cases, vascular abnormalities may be secondary to abnormal hemodynamics created by a faulty aortic valve. However, multiple studies have demonstrated aortic dissections and aneurysms in patients without hypertension, hemodynamic perturbations, or with only mildly abnormal valves, suggesting an intrinsic defect in the aorta of these patients $(26,30,48,49)$. Interestingly, evidence of cystic medial necrosis, a process in which aortic vascular smooth muscle undergoes apoptosis, has been found in patients before dilation of the aorta is clinically noted (28). In addition, evidence of fragmented elastic fibers with greater distance between fibers has been reported (31). The contribution of neural crest to the smooth muscle of the ascending aorta and aortic arch is well documented $(7,10)$, and our neural crest and Notch mutants reveal disorganized aortic intimal layers with increased distance between cells (Supplemental Figure 4 and ref. 45). To the best of our knowledge, our data provide the first experimental evidence unifying the developmental mechanisms underlying semilunar valve and aortic arch abnormalities.

TGF- $\beta$ signaling has been extensively studied in the setting of aortic aneurysm formation, Marfan syndrome, and related disorders (50). Marfan syndrome is a disease characterized by mutations in FIBRILLIN1, which usually sequesters TFG- $\beta$. Without this modulation, an increase of TGF- $\beta$ signaling manifests as aortic root dissection, skeletal overgrowth, pulmonary emphysema, and ocular lens dislocation, the classic signs of Marfan syndrome (50). Loeys-Dietz syndrome is an autosomal dominant syndrome closely related to Marfan syndrome, characterized by gain-of-function mutations in the genes encoding the type I or II TGF- $\beta$ receptor, arterial tortuosity, arterial dissections and aneurysms, bicuspid aortic valve, and various craniofacial abnormalities (50-52). Importantly, the aortas of Marfan syndrome patients and patients with bicuspid aortic valve and aortic aneurysm demonstrate loss of elastic fiber architecture and abnormal distribution of collagen and other extracellular matrix proteins $(53,54)$. Vascular specimens from Loeys-Dietz patients and nonsyndromic cases of patients with bicuspid aortic valve and aortic aneurysm demonstrate increased TGF- $\beta$ signaling. Reduction of TGF- $\beta$ signaling through the administration of neutralizing antibodies or chemical antagonists can attenuate the phenotypes $(51,52,54-56)$. Notch and TGF- $\beta$ signaling have been shown to cooperate to promote vascular smooth muscle differentiation (57), although we were unable to detect increases in phospho-Smad2/3 in our Notch and neural crest mutants (data not shown). Further studies will be needed to elucidate the role of TGF- $\beta$ signaling in the tissue-tissue interactions that we have described during aortic arch and semilunar valve remodeling.

As the neural crest migrates through the pharyngeal mesenchyme, it is in close proximity to the second heart field, a likely location for signaling between these 2 cell populations (Figure 3, $\mathrm{F}$ and $\mathrm{G})$. Bmp 4 is robustly expressed in the 2 columns of cardiac neural crest as they enter the outflow tract and populate the endocardial cushions (15) and is a mediator of apoptosis in other tissues (58). Bmp4 expression in cardiac neural crest is diminished upon Notch inhibition in the second heart field (15). Hence, Bmp4 is an attractive candidate as a mediator of apoptotic signaling from neural crest that can respond to signals from the second heart field (15). Indeed, Bmp4 is required for proper aortic arch remodeling and semilunar valve development $(59,60)$. However, deletion of Bmp4 in the neural crest (using Wnt1-cre) does not result in abnormal semilunar valves (data not shown). Msx signaling has been shown to be an important regulator of neural crest apoptosis (61), and Msx genes can function downstream of Bmp signaling. However, we have not observed consistent changes in Msx1 or Msx2 expression in late 
gestation Notch mutant valves (data not shown). Finally, MMP signaling has been the focus of intense investigation in its role in bicuspid aortic valve and aortic aneurysm formation and progression. Specifically, MMP-2 and MMP-9 levels are altered in in vitro and ex vivo studies of bicuspid aortic valve and aneurysms $(53,62,63)$. However, it must be elucidated whether abnormal levels of MMP-2 or MMP-9 are causal in the phenotype or a marker of disease. In addition, deciphering the spatial and temporal expression of MMP signaling during semilunar valve development will be of great interest. The identification of the neural crest-derived apoptotic signal or signals that are required for semilunar valve remodeling will be the focus of future studies, as will the characterization of signals responsible for altered extracellular matrix production.

In summary, our data suggest that neural crest provides an instructive signal for remodeling of the semilunar valves and highlight the importance of tissue-tissue interactions among second heart field, neural crest, and endocardial cushion mesenchyme. The experimental demonstration of a role for neural crest in the pathophysiology of congenital semilunar valve disorders provides a developmental mechanism to explain the association of aortic and pulmonary valve defects with vascular abnormalities of the aortic arch.

\section{Methods}

Mice. Pax $3^{\mathrm{Cre} /{ }^{+}}$mice were outbred onto a mixed CD1 background. Pax3 ${ }^{\mathrm{Cre}}$, Islet $1^{\text {Cre }}$, Mef2c-AHF-Cre, and DNMAML mice were genotyped as previously described (40). Littermate animals were compared in all experiments unless otherwise noted. All animal protocols were approved by the University of Pennsylvania Institutional Animal Care and Use Committee. James F. Martin (Texas A\&M University, College Station, Texas, USA) provided Wnt1-cre;Bmp $4^{\text {flox/flox }}$ embryos.

Histology, immunobistochemistry, and in situ bybridization. Samples were fixed overnight (E9.5 to E11.5 embryos) or for 48 hours (E16.5 to E17.5 embryos) with 4\% paraformaldehyde and dehydrated through an ethanol series. Samples were then paraffin embedded and sectioned. Antibodies used for immunostaining were mouse monoclonal antiAP2 $\alpha$ (catalog \#5E4; Developmental Studies Hybridoma Bank), rabbit polyclonal anti-GFP (catalog A11122; Invitrogen), and mouse monoclonal anti-phospho-histone H3 (catalog 9706L; Cell Signaling Technology). TUNEL staining was performed using standard protocols. Radioactive in situ hybridizations were performed using previously described probes for Sema3C (38) and PlexinA2 (15). Immunohistochemistry and in situ hybridization images were analyzed using Adobe Photoshop. Brightness and contrast were altered identically in control and mutant images in all instances using Adobe Photoshop.
H\&E and Modified Movat's Pentachrome staining were performed using standard protocols. Quantification of phospho-histone- $\mathrm{H}^{+}, \mathrm{DAPI}^{+}$, and TUNEL $^{+}$cells was performed using Metamorph software. Triple filter images were used in the analysis to exclude nonspecific signal, and leaflets were traced from images in a manner blinded to genotype.

Intrauterine echocardiography. Pregnant female mice were anesthetized by inhalation of $2 \%$ isoflurane in a glass chamber and $1 \%$ to $1.5 \%$ isoflurane via nose cone to maintain anesthesia. The mice were imaged on a heated platform while monitoring body temperature. Echocardiography was performed using a Vevo 770 (VisualSonics) with a linear 30-MHz probe (RMV 707). A map of the position of the embryos, constructed during each study, was used to dissect the embryos at the conclusion of the study for genotyping. Pulse-wave Doppler analysis of the aorta was used to assess aortic insufficiency.

$O P T$. Embryos were harvested into cold PBS, and the heart and great arteries were dissected. The organs were fixed overnight in $4 \%$ paraformaldehyde. Organs were embedded in $1 \%$ low-melt agarose, dehydrated in methanol, and then cleared in 1:2 (v/v) benzyl alcohol and benzyl benzoate (64). Organs were then scanned using the Bioptonics OPT Scanner (3001M). The semilunar valves and leaflets were outlined in Adobe Photoshop, and image stacks were reconstructed using OsiriX software. Volumes of reconstructed images of leaflets were calculated using Volocity software.

Statistics. Students' 2-tailed $t$ test analysis was used to evaluate differences between groups. $P<0.05$ was considered statistically significant. OPT quantitative data and proliferation indices are represented as average \pm SEM.

\section{Acknowledgments}

We thank Arun Padmanabhan, Nikhil Singh, and the rest of the Epstein laboratory for many helpful discussions. We thank Nicole Antonucci and Ashley Cohen for assistance with animal husbandry and thank James F. Martin for sharing specimens of Wnt1-cre;Bmp $4^{f l o x} / f l o x$ embryos. This work was supported by an American Heart Association Physician-Scientist/Post-Doctoral Fellowship (AHA 0825548D to R. Jain); the University of Pennsylvania, Division of Cardiology T-32 and Benjamin \& Mary Siddons Measey Foundation (to S. Rentschler), and funds from the AHA DeHaan Myogenesis Center, the NIH(U01 HL100405, R01 HL095634), and the WW Smith Endowed Chair for Cardiovascular Research (to J.A. Epstein).

Received for publication July 5, 2010, and accepted in revised form November 1, 2010.

Address correspondence to: Jonathan A. Epstein, 1154 BRB II, 421 Curie Blvd., Philadelphia, Pennsylvania 19104, USA. Phone: 215.898.8731: Fax: 215.573.2094; E-mail: epsteinj@mail.med. upenn.edu.
1. Nakajima Y, Yamagishi T, Hokari S, Nakamura H. Mechanisms involved in valvuloseptal endocardial cushion formation in early cardiogenesis: roles of transforming growth factor (TGF)-beta and bone morphogenetic protein (BMP). Anat Rec. 2000;258(2):119-127.

2. Butcher JT, Markwald RR. Valvulogenesis: the moving target. Philos Trans R Soc Lond B Biol Sci. 2007;362(1484):1489-1503

3. Combs MD, Yutzey KE. Heart valve development: regulatory networks in development and disease. Circ Res. 2009;105(5):408-421.

4. Markwald RR, Norris RA, Moreno-Rodriguez R, Levine RA. Developmental basis of adult cardiovascular diseases: valvular heart diseases. Ann NY Acad Sci. 2010;1188:177-183.

5. Fedak PW, Verma S, David TE, Leask RL, Weisel
RD, Butany J. Clinical and pathophysiological implications of a bicuspid aortic valve. Circulation. 2002;106(8):900-904.

6. Jain R, Rentschler S, Epstein JA. Notch and cardiac outflow tract development. Ann N Y Acad Sci. 2010;1188:184-190.

7. Kirby ML, Gale TF, Stewart DE. Neural crest cells contribute to normal aorticopulmonary septation. Science. 1983;220(4601):1059-1061.

8. Stoller JZ, Epstein JA. Cardiac neural crest. Semin Cell Dev Biol. 2005;16(6):704-715.

9. High FA, et al. An essential role for Notch in neural crest during cardiovascular development and smooth muscle differentiation. J Clin Invest. 2007; 117(2):353-363.

10. Jiang X, Rowitch DH, Soriano P, McMahon AP, Sucov HM. Fate of the mammalian cardiac neural crest. Development. 2000;127(8):1607-1616.

11. Gitler AD, Brown CB, Kochilas L, Li J, Epstein JA. Neural crest migration and mouse models of congenital heart disease. Cold Spring Harb Symp Quant Biol. 2002;67:57-62.

12. Snarr BS, Kern CB, Wessels A. Origin and fate of cardiac mesenchyme. Dev Dyn. 2008;237(10):2804-2819.

13. Verzi MP, McCulley DJ, De Val S, Dodou E, Black $\mathrm{BL}$. The right ventricle, outflow tract, and ventricular septum comprise a restricted expression domain within the secondary/anterior heart field. Dev Biol. 2005;287(1):134-145.

14. Cai CL, et al. Isl1 Identifies a cardiac progenitor population that proliferates prior to differentiation and contributes a majority of cells to the heart. Developmental Cell. 2003;5(6):877-889.

15. High FA, et al. Murine Jagged1/Notch signaling in 
the second heart field orchestrates Fgf8 expression and tissue-tissue interactions during outflow tract development. J Clin Invest. 2009;119(7):1986-1996.

16. $\mathrm{Li} \mathrm{L}$, et al. Alagille syndrome is caused by mutations in human Jagged 1, which encodes a ligand for Notch1. Nat Genet. 1997;16(3):243-251.

17. Oda $\mathrm{T}$, et al. Mutations in the human Jagged 1 gene are responsible for Alagille syndrome. Nat Genet. 1997;16(3):235-242.

18. Krantz ID, Piccoli DA, Spinner NB. Clinical and molecular genetics of Alagille syndrome. Curr Opin Pediatr. 1999;11(6):558-564

19. Greenway SC, et al. De novo copy number variants identify new genes and loci in isolated sporadic tetralogy of Fallot. Nat Genet. 2009;41(8):931-935.

20. Bauer RC, et al. Jagged1 (JAG1) mutations in patients with tetralogy of Fallot or pulmonic stenosis. Hum Mutat. 2010;31(5):594-601.

21. Garg V, et al. Mutations in NOTCH1 cause aortic valve disease. Nature. 2005;437(7056):270.

22. Mohamed SA, et al. Novel missense mutations (p.T596M and p.P1797H) in NOTCH1 in patients with bicuspid aortic valve. Biochem Biophys Res Commun. 2006;345(4):1460-1465.

23. McKellar SH, Tester DJ, Yagubyan M, Majumdar R, Ackerman MJ, Sundt TM 3rd. Novel NOTCH1 mutations in patients with bicuspid aortic valve disease and thoracic aortic aneurysms. J Thorac Cardiovasc Surg. 2007;134(2):290-296.

24. Hoffman JIE, Kaplan S. The incidence of congenital heart disease. J Am Coll Cardiol. 2002; 39(12):1890-1900

25. Kappetein AP, Gittenberger-de Groot AC, Zwinderman AH, Rohmer J, Poelmann RE, Huysmans HA. The neural crest as a possible pathogenetic factor in coarctation of the aorta and bicuspid aortic valve. J Thorac Cardiovasc Surg. 1991;102(6):830-836.

26. Schievink WI, Mokri B. Familial aorto-cervicocephalic arterial dissections and congenitally bicuspid aortic valve. Stroke. 1995;26(10):1935-1940.

27. Arrington CB, et al. Absence of TGFBR1 and TGFBR2 mutations in patients with bicuspid aortic valve and aortic dilation. Am J Cardiol. 2008; 102(5):629-631.

28. Bonderman D, Gharehbaghi-Schnell E, Wollenek G, Maurer G, Baumgartner H, Lang IM. Mechanisms underlying aortic dilatation in congenital aortic valve malformation. Circulation. 1999;99(16):2138-2143.

29. de Sa M, Moshkovitz Y, Butany J, David TE. Histologic abnormalities of the ascending aorta and pulmonary trunk in patients with bicuspid aortic valve disease: clinical relevance to the ross procedure. J Thorac Cardiovasc Surg. 1999;118(4):588-594.

30. Tadros TM, Klein MD, Shapira OM. Ascending aortic dilatation associated with bicuspid aortic valve: pathophysiology, molecular biology, and clinical implications. Circulation. 2009;119(6):880-890.

31. Jain D, Dietz HC, Oswald GL, Maleszewski JJ, Halushka MK. Causes and histopathology of ascending aortic disease in children and young adults [published online ahead of print November 18, 2009]. Cardiovasc Pathol. doi:10.1016/ j.carpath.2009.09.008.

32. Chalepakis G, Goulding M, Read A, Strachan T, Gruss P. Molecular basis of splotch and Waarden- burg Pax-3 mutations. Proc Natl Acad Sci U S A. 1994;91(9):3685-3689.

33. Goulding MD, Chalepakis G, Deutsch U, Erselius JR, Gruss P. Pax-3, a novel murine DNA binding protein expressed during early neurogenesis. $E M B O J$. 1991;10(5):1135-1147.

34. Conway SJ, Bundy J, Chen J, Dickman E, Rogers R, Will BM. Decreased neural crest stem cell expansion is responsible for the conotruncal heart defects within the splotch $(\mathrm{Sp}(2 \mathrm{H})) / \mathrm{Pax} 3$ mouse mutant. Cardiovasc Res. 2000;47(2):314-328.

35. Epstein JA. Pax3, neural crest and cardiovascular development. Trends Cardiovasc Med. 1996;6(8):255-260.

36. Epstein JA, et al. Migration of cardiac neural crest cells in Splotch embryos. Development. 2000; 127(9):1869-1878.

37. Schaefer BM, et al. The bicuspid aortic valve: an integrated phenotypic classification of leaflet morphology and aortic root shape. Heart. 2008; 94(12):1634-1638

38. Gitler AD, Lu MM, Epstein JA. PlexinD1 and Semaphorin Signaling are Required in Endothelial Cells for Cardiovascular Development. Dev Cell. 2004;7(1):107-116

39. Brown CB, et al. PlexinA2 and semaphorin signaling during cardiac neural crest development. Development. 2001;128(16):3071-3080.

40. Engleka KA, Gitler AD, Zhang M, Zhou DD, High FA, Epstein JA. Insertion of Cre into the Pax3 locus creates a new allele of Splotch and identifies unexpected Pax3 derivatives. Dev Biol. 2005;280(2):396-406.

41. Tu L, et al. Notch signaling is an important regulator of type 2 immunity. J Exp Med. 2005 202(8):1037-1042.

42. Novak A, Guo C, Yang W, Nagy A, Lobe CG. Z/ EG, a double reporter mouse line that expresses enhanced green fluorescent protein upon Cremediated excision. Genesis. 2000;28(3-4):147-155.

43. Timmerman LA, et al. Notch promotes epithelialmesenchymal transition during cardiac development and oncogenic transformation. Genes Dev. 2004;18(1):99-115.

44. Macatee TL, Hammond BP, Arenkiel BR, Francis L, Frank DU, Moon AM. Ablation of specific expression domains reveals discrete functions of ectoderm- and endoderm-derived FGF8 during cardiovascular and pharyngeal development. Development. 2003;130(25):6361-6374.

45. Brown CB, Wenning JM, Lu MM, Epstein DJ, Meyers EN, Epstein JA. Cre-mediated excision of Fgf8 in the Tbx1 expression domain reveals a critica role for Fgf8 in cardiovascular development in the mouse. Dev Biol. 2004;267(1):190-202.

46. Park EJ, et al. An FGF autocrine loop initiated in second heart field mesoderm regulates morphogenesis at the arterial pole of the heart. Development. 2008;135(21):3599-3610.

47. Oliver JM, et al. Risk of aortic root or ascending aorta complications in patients with bicuspid aortic valve with and without coarctation of the aorta. Am J Cardiol. 2009;104(7):1001-1006.

48. Pachulski RT, Weinberg AL, Chan KL. Aortic aneurysm in patients with functionally normal or minimally stenotic bicuspid aortic valve. Am J Cardiol. 1991;67(8):781-782.
49. Hahn RT, Roman MJ, Mogtader AH, Devereux RB. Association of aortic dilation with regurgitant, stenotic and functionally normal bicuspid aortic valves. J Am Coll Cardiol. 1992;19(2):283-288.

50. Ramirez F, Dietz HC. Marfan syndrome: from molecular pathogenesis to clinical treatment. Curr Opin Genet Dev. 2007;17(3):252-258.

51 . Loeys BL, et al. A syndrome of altered cardiovascular, craniofacial, neurocognitive and skeletal development caused by mutations in TGFBR1 or TGFBR2. Nat Genet. 2005;37(3):275-281.

52 . Loeys BL, et al. Aneurysm syndromes caused by mutations in the TGF-beta receptor. N Engl J Med. 2006; 355(8):788-798.

53. Nataatmadja $M$, et al. Abnormal extracellular matrix protein transport associated with increased apoptosis of vascular smooth muscle cells in marfan syndrome and bicuspid aortic valve thoracic aortic aneurysm. Circulation. 2003;108 suppl 1:II329-II334.

54. Habashi JP, et al. Losartan, an AT1 antagonist, prevents aortic aneurysm in a mouse model of Marfan syndrome. Science. 2006;312(5770):117-121.

55. Gomez D, et al. Syndromic and non-syndromic aneurysms of the human ascending aorta share activation of the Smad2 pathway. J Pathol. 2009;218(1):131-142.

56. Maleszewski JJ, Miller DV, Lu J, Dietz HC, Halushka MK. Histopathologic findings in ascending aortas from individuals with Loeys-Dietz syndrome (LDS). Am J Surg Pathol. 2009;33(2):194-201.

57. Tang Y, et al. Notch and transforming growth factor-beta (TGFbeta) signaling pathways cooperatively regulate vascular smooth muscle cell differentiation. J Biol Chem. 2010;285(23):17556-17563.

58. Graham A, Francis-West P, Brickell P, Lumsden A. The signalling molecule BMP4 mediates apoptosis in the rhombencephalic neural crest. Nature. 1994;372(6507):684-686

59. Liu W, et al. Bmp4 signaling is required for outflowtract septation and branchial-arch artery remodeling. Proc Natl Acad Sci U S A. 2004;101(13):4489-4494.

60. McCulley DJ, Kang JO, Martin JF, Black BL. BMP4 is required in the anterior heart field and its derivatives for endocardial cushion remodeling, outflow tract septation, and semilunar valve development. Developmental Dynamics. 2008; 237(11):3200-3209.

61. Tribulo C, Aybar MJ, Sanchez SS, Mayor R. A balance between the anti-apoptotic activity of Slug and the apoptotic activity of $\mathrm{msx} 1$ is required for the proper development of the neural crest. Dev Biol. 2004;275(2):325-342.

62. Koullias GJ, Korkolis DP, Ravichandran P, Psyrri A, Hatzaras I, Elefteriades JA. Tissue microarray detection of matrix metalloproteinases, in diseased tricuspid and bicuspid aortic valves with or without pathology of the ascending aorta. EurJ Cardiothorac Surg. 2004;26(6):1098-1103.

63. Segura AM, et al. Immunohistochemistry of matrix metalloproteinases and their inhibitors in thoracic aortic aneurysms and aortic valves of patients with Marfan's syndrome. Circulation. 1998; 98(19 suppl):II331-II337.

64. Sharpe J, et al. Optical projection tomography as a tool for 3D microscopy and gene expression studies. Science. 2002;296(5567):541-545 